\title{
Trend-Casting in the Interactive Digital Media Industry : Some Results and Guidelines
}

\author{
Ravi S. Sharma*, Yang $\mathbf{Y i}^{* *}$
}

Submitted: January 30, 2013; Accepted: April 1, 2013; Published Online: April 12, 2013

\begin{abstract}
In this practice article, we present the results of a scenario planning approach that is a hybrid of the three main schools of thought. Our research objective was to study the future of Interactive Digital Media applications such as online music, on-demand television and massively multi-player online role-playing games. Our approach, while essentially qualitative in nature, nevertheless draws from the rigors of the quantitative school in identifying and then tracking the significant dimensions of analysis that emerge over time as strands of events leading to plausible scenarios. Our empirical analysis revealed mapping strands to three themes - ownership, distribution and innovation - which we used in an expert validation exercise to formulate scenarios. We present and discuss the major findings and implications of this empirical investigation. In a nutshell, we conjecture that an open, competitive IDM marketplace with performance safeguards may serve both and lead to a win-win scenario. While there are differences among IDM sectors, a unified approach to regulation and policy would be effective.
\end{abstract}

Keywords Digital media technology, strategic industry analysis, digital economy

\section{Introduction}

Innovation and social change brought about by the Internet is set to transform traditional media through a more efficient production, new forms of distribution, the entry of new players and much greater choice for consumers (Boaventura \& Fischmann 2008; Castronova, 2005). The convergence processes characterizing the multimedia age and leading towards the information society have led to a

\footnotetext{
* Wee Kim Wee School of Communication \& Information, Nanyang Technological University, 31 Nanyang Link, Singapore 637718; rsharma@pmail.ntu.edu.sg

** Thomson Reuters Asia, 18 Science Park Drive, Singapore 118229; yangyijane@gmail. com
} 
deep transformation of how and when media is consumed. Such e-businesses are in fact being shaped by a number of driving forces such as the digitalization of the traditional content, the diffusion of new alternative access technologies, the developments in interactive technologies, and greater accessibility of technology (Berman, 2007; Ritchey, 2002; Swatman, Krueger, $\&$ van der Heijden, 2006). The Interactive Digital Media (IDM) marketplace includes a host of services such as music, video, games, books, software and others over broadband and web interfaces to a variety of devices from smartphones to home theatre systems, through which users communities interact with application and themselves. For the purpose of this research, the scope of the IDM industry includes online music, Internet Protocol TV (IPTV) and massive multi-player role players game (MMORPG).

It is generally argued that the traditional media industry has changed significantly and fundamentally over the past decade with new entrants bringing fresh opportunities and emerging competition (Dong, 2007; Szulansk and Amin, 2004). For instance, Internet Service Providers offer existing media companies multiple opportunities to aggregate, syndicate or distribute their business in this innovative era while them able to offer content over the network. Customers play a more significant role in this business. Given unprecedented choices of new technologies, they become highly discerning and interactive (Spero \& Stone, 2004). The rich consumer selection and gap between production hype and market penetration jointly shape the future trend of content consumption in the digital age. However, the extent to which this will actually happen, how quickly it will happen, and how it will affect specific markets, are all unclear at this stage. This is the context of the research reported in this paper.

In this paper, we provide a narrative of major tensions within the IDM industry and triggers for its future development. From this we try to seek a feasible and sustainable business scenario for the IDM industry. Specifically, we explore whether there is a win - win scenario for all stakeholders. If so, how do we bridge the gap to achieve that scenario? It is the intended contribution of this paper that industry practitioners get such a view for growth and development. The remainder of paper includes a brief introduction of the empirical approach in the next section. Section 3 is about the results and analysis that are derived by this empirical approach. Section 4 discusses the potential scenarios and respective policy implications. The paper concludes by drawing insights on what these scenarios mean for the IDM sectors studied below. 


\section{Empirical Approach}

A number of scholars have explored several IDM sectors (cf. Bouwman et al. 2008; Coles et al. 2004; Griffith \& Taylor, 1996; Poel et al 2007; Shin, 2005; Swatman et al., 2006), with a specific focus on the micro aspect of an industry's respective development with respect to political, economic, social, or technological factors. However, little scholarly attention has been paid to the growth from a macro and futuristic perspective that analyzes the trends and direction of IDM development ahead. This paper has observed the holistic trends and market development through a synthesized environmental scan. The approach adopted in this empirical study has been used to formulate from the events and triggers, some key trends and patterns in IDM sector across sectors, and sources and time periods. Fundamentally, the concept of Scenario Planning (SP) is based on the notion that the future is unpredictable. The main purpose of scenario building is to construct several different alternative futures and the respective paths that lead to them (Georgantza and Acar, 1995; Millett, 2003; Ogilvy, 2002). Among numerous techniques and methods of SP, the synthesized approach conducted in this research is the result of comparison and contrast of the Shell approach (Cornelius, et al, 2005), French method-logy (Godet, 2006) and Firecracker method (Pereira, 2004).

The following is a brief introduction to our empirical approach. First, an extensive literature review and in-depth Delphi interview will support the validity for the subsequent search and tracking. Second, with aid of automated tracking software, the latest news from authoritative sources and popular theme blogs will be gathered as events / triggers. These events / triggers will compose strands, which may morph to formulate different scenarios. Finally, an expert workshop will be held to validate scenarios and prescribe a possible win-win scenario, and offer strategic planning support. Each of these steps of the synthesized approach has been described in detail in Yang and Sharma (forthcoming). The synthesized approach is not claimed as the best scenario planning method; however it possesses greater applicability in the field of research of this study.

More specifically, our SP approach began with an environmental analysis based on common socio-economic categories (Guion 2010). Prior to that, a survey of the technical literature on the 3 IDM sectors of interest (online music, MMORPG, and on-demand TV) was conducted to overcome the subjectivity and arbitrariness inherent in the main approaches. In order to be comprehensive, the literature review should focus on four perspectives - legal, economic, social and technological (the LEST framework). The aim of this stage is to derive the disruptions, which affect the development of the respective IDM sectors. This will also help track events and triggers and formulate scenarios in the next stages. Table 1 shows the outcome of applying 
Steps 1 and 2 of the hybrid SP approach to the three IDM sectors of interest. Significant factors, repeatedly mentioned in the literature as determinants of success, were selected as Dimensions of Analysis (DOAs).

Table 1 Construction of data dictionary for tracking of triggers

$\mathrm{IDM}=($ online music + internet music + web music + MP3 content + virtual music store $)$

OR (Internet TV + IPTV + time-shifted TV + mobile TV + on-demand TV + web TV)

OR (MMORPG + virtual worlds + online games + internet games + cyber games)

Content $=$ censorship $\{$ strong vs. weak $\} 11+$ ownership $\{$ enforceable vs. unenforceable + viewership \{in the room vs. on the move $\}+$ storage \{encrypted vs. unencrypted + consumption \{downloading vs. streaming

Platforms $=$ standards $\{$ regulated vs. unregulated $\}+$ commercialization $\{$ proprietary vs. licensed $\}+$ access devices $\{$ wired vs. wireless $\}+$ architecture $\{$ open vs. closed $\}+$ internet penetration \{low vs. high

Distribution $=$ market position $($ entry vs. growth $)+$ channel $\{$ direct vs. aggregated $\}+$ payment $\{$ per view vs. subscription $\}+$ role $\{$ consumer vs. prosumer $\}+$ referrals \{recommendation vs. searching\}

Innovation $=$ network policy $\{$ net neutrality vs. segmented $\}$ +business model $\{$ two sided markets vs. one sided market $\}+$ e-word of mouth (effective vs. ineffective) + end user design (customized vs. non-customized)

IPR = IP law \{strong vs. weak $\}+$ IP licensing \{enforceable vs. unenforceable $\}+$ Respect for IP \{high vs. low + DRM \{loose vs. tight $\}$

Consumer Behavior $=$ conditional access $\{$ restricted vs. unrestricted $\}+$ market trends \{growth vs. mature $\}+$ social networks $\{$ long vs. short tailed $\}+$ market chasm $\{$ early vs. late adoption $\}+$ switching cost $\{$ high vs. low $\}$

The Dimensions of Analysis have been formulated under the LEST framework that we used for scenario planning. Each Dimension of Analysis represents one key disruption. For instance, shift and transformation of Platform is under the Economic issues, while Consumer Behavior is mainly influenced by issues of social perspectives. The tension points of each Dimension of Analysis are built with the consensus among groups of experts via the Delphi interview. The Dimensions of Analysis (shown in bold in Table 1) were next codified using web-crawling software, to track the three IDM sectors for a 6-month period in order to consistently discern patterns or trends that contribute to a likely future state. Also shown are Tension Points (in italics) that calibrate the range of possibilities for a given Dimension of Analysis to be tracked so as to analyze the plausibility of various scenarios to be developed. A high level overview of the hybrid approach is as follows.

As well, the Dimensions of Analysis and Tension Points were derived by first unearthing critical success factors from the research literature and then 
through seeking consensus from the Delphi panel as described in Steps 1 and 2. The final data dictionary that was used for the next stages is that one shown in Table 1. Steps 3 and 4 of the synthesized approach required extensive use of a customizable, online, easy-to-use tool known as Trendicate (www.nexlabs. com). Its functionalities include the definition of the data dictionary, training the tracking system with feedback on relevance, and validating the automated tracking with manual checks using content analysis techniques. The development and use of the analytic tools is beyond the scope of this thesis. Suffice to say that using the data dictionary derived above, Trendicate tracks a given list of online sources (in this case online news and blogs such as New York Times, Financial Times, the Straits Times, and etc.) and produces a matrix of frequencies of such "triggers".

More specifically, Trendicate allows the tracking of websites and blogs for the occurrence and co-occurrence of keywords of interest. It does so by tracking and logging various Internet sites (including blogs) for a set of keywords. At regular intervals, the system may be configured to produce reports of the frequencies of the tracked keywords as well as summaries of source documents. Hence, based on the general literature search and consensus among Delphi experts, Dimensions of Analysis and Tension Points (shown in Table 1) have been entered to train the system and track the relevant counts for triggers or events (that is, mainstream news and blog articles from the abovementioned sources). In this case, triggers are the occurrence of keywords such as Dimensions of Analysis, Tension Points and their synonyms. It is worth mentioning at this point that the tracking involved a limited but intensive training period.

We apply the steps outlined above on the online music, IPTV and MMORPG sectors to project the future development trends of each. From which a feasible and sustainable scenario may be built and subsequent policy implications and suggestions will be proposed accordingly.

\section{Results and Analysis}

An analysis of frequency and dependency ratio matrices is performed as step four of the synthesized Scenario Planning. Three strands or patterns may be extracted which are labeled as - ownership, distribution and innovation. It is apparent that these are the tensions between the two prominent players in the IDM marketplace, namely the producers and the consumers.

Analyzing the key trends, a general key conclusion is that regardless of specific sector, there are significant tensions between suppliers (content providers as well as technology providers) and consumers. This was repeatedly 
clear along several Dimension of Analysis as shown in the previous sections. The tabulated findings were derived from the strands formulated by Trendicate and validated during an expert validation workshop that is the final step of the SP approach. This was conducted in a workshop format by the authors. It involved presenting the key themes derived from news sources and asking for comments and discussion.

This schism between producers and consumers along the dimensions listed in Table 2 is critical to note. Mostly, they corroborate previous research. Some are synergistic, others are complementary and strands or patterns emerged to help formulate win-win or alternate scenarios. The three strands identified above form the cornerstone of the findings and shall be discussed later in this section.

Table 2 Inter-play between producers and consumers

\begin{tabular}{|l|l|}
\hline \multicolumn{1}{|c|}{ Providers } & \multicolumn{1}{c|}{ Consumers } \\
\hline $\begin{array}{l}\text { DRM and IP law are the key to enforcing } \\
\text { ownership of digital content (IFPI, 2005b, } \\
\text { 2008). }\end{array}$ & $\begin{array}{l}\text { Regulation (including censorship and } \\
\text { rights) are key factors influencing } \\
\text { consumption (Griffs \& Taylor, 1996). }\end{array}$ \\
\hline $\begin{array}{l}\text { Top-down, closed architecture is the } \\
\text { preferred means of distribution. The } \\
\text { distribution channel is a discovery process } \\
\text { involving search and referrals (Karakas, } \\
\text { 2009). }\end{array}$ & $\begin{array}{l}\text { End-user design is the preferred mode of } \\
\text { customising applications and their usage. } \\
\text { This includes choice of network, device } \\
\text { and, above all, place and time (Hsu \& Lu, } \\
\text { 20o). }\end{array}$ \\
\hline $\begin{array}{l}\text { Innovation such as multi-homing, content } \\
\text { repurposing and immersive interaction } \\
\text { offer novelty and convenience to audiences } \\
\text { (Lucchi, 2007). }\end{array}$ & $\begin{array}{l}\text { eWom, social networks, viral marketing } \\
\text { and prosumer content are user-driven } \\
\text { means of innovation and promoting } \\
\text { consumption (Molteni \& Ordanini, 2003; } \\
\text { Pagani, 20o9). }\end{array}$ \\
\hline
\end{tabular}

Another conclusion that emerged was that the new entrants to this emerging industry bring not only potential cooperation opportunities but also conflicts of interest and latent regulatory uncertainties. Although from a macroperspective, the three IDM sectors are quite distinct from each other, they share several intrinsic similarities and encounter challenges alike as observed from the Mantel tests and RMDS analyses. Based on these complex and dynamic situations, there are also cautionary factors that should be taken into consideration in scenario construction.

The third conclusion is that the self-regulated respect for the IP Tension Point does not exist in all three sectors despite the fact that Intellectual Property Rights play a pivotal role. It is echoed in previous studies about the free web economy and consequent shift of consumer psyche (Coles et al, 2004; 
Duchene \& Waelbroeck, 2006; IFPI, 2005a, 2008). Equipped with the social network of sharing, potential consumers feel entitled to share digital products for a number of varied reasons, regardless of the IP regime. This requires more appropriate legal and technological counter-measures to resolve this issue. A changing business model plausibly is an integral part of resolving the problem. Apple's walled-garden (Berman et al 2007) iTunes is a good example of a business model for digital music that circumvents the problem of piracy. For IPTV, the IP protection of premium content and avatars are always concerns that remain to be resolved satisfactorily.

\section{Discussion of Implications}

Earlier in this paper, it was suggested that three categories of scenarios could be derived from the hybrid approach to SP:

1. Win-Win Scenarios (a fair and sustainable growth regime where all the players gain from an enlarged market size),

2. Win-Lose \& Lose-Win Scenarios (an unduly competitive, winner-takes-all, zero-sum game regime where the market does not grow and is re-apportioned among the players),

3. Lose-Lose scenarios (reduction to a classic Prisoners' Dilemma regime).

A win-win scenario is defined as a circumstance in which all the players (mainly the suppliers and consumers) are in harmonious relations, and gain what they reasonably expect from the market. In other words, they would be no worse off than in other less optimal scenarios. Even when an industry is in a sustainable development phase and may not necessarily grow very fast, it must be profitable from industry's perspective. Otherwise, we picture the alternate scenarios as win-lose or lose-win scenarios (where either producers or consumers lose), which are neither sustainable nor desirable for the growth of the sector or the IDM industry as a whole.

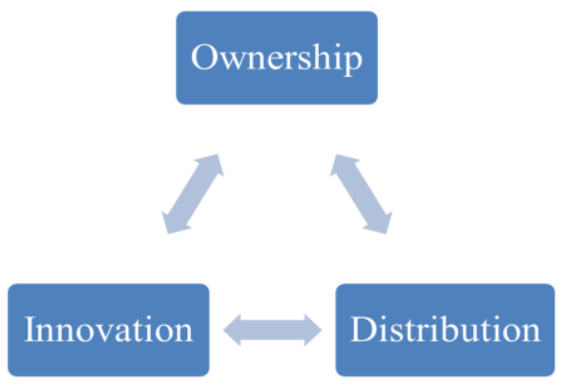

Figure 1 Policy parameters for scenario construction 
Figure 1 depicts the three themes that were derived from the analysis of the empirical investigations. In the context of the research, ownership refers to the control and exploitation of digital content; innovation refers to the (co-) creation of applications and services; and, distribution refers to the right to provide consumer access to such IDM applications and bill for them. These three themes may be considered policy parameters that interpolate to influence win-win or alternate outcomes. Based on the three identified strands or patterns, it may be generalized that the policy contentions among them need to be put into consideration for scenario building. From the scenario validation workshop, several qualitative anecdotal illustrations also emerged which were used in our recommendations.

Based on the analysis and findings of the research, we suggest the following policy implications for policy makers who make laws and frameworks governing the IDM marketplace and industry regulators who enforce such laws in order to promote jurisdictional objectives.

\section{Ownership: Intellectual Property Rights as a Basis for Growth and Sustainability}

First, it should be accepted that in the globalized economy, and especially on the Internet, there are rarely globally enforceable laws to protect consumer rights (Berman, 2007; Castronova, 2005a; Fabbe et al, 2011). This is difficult if not impossible, due to the diversified social and political systems. Under these circumstances, with the phenomenal technological development and economic transformation, a regulator can no longer keep a market under control. It requires the cooperation of the other stakeholders.

Second, now that the role of consumers has transferred from a passive receiver in the past to a proactive participant, there is a need to reconsider digital and intellectual property within an IDM industry framework. The interaction-empowered consumers enjoy freedom they never enjoyed before. However, there is lack of legal ways to define their roles and regulate their activities. With technological uncertainties coupled with IDM industry development poised to continue, the initial balance between consumers and industry players established by various copyright laws has been jeopardized (Lucchi, 2007).

Further, intellectual property owners have never held an unlimited control over the use of their works: in effect. Copyright and related rights are a legal concept that contains several provisions that limit the monopoly control granted to right holders (McCaslin and Burross, 2011). On the other hand, the high literacy about new media and technologies among current consumers 
enables the violation of copyright to be easy and pervasive. This contention will exist for the near future. Thus, there is a problem that traditional IP laws and regulations are generated to protect a digital monopoly rather than the consumers' right to access the IP. However, the current new technologies and open access platforms (essentially Internet based) empower consumers to share freely and become part of the distribution chain, which damage IP owners' (the industry) interest. Whereas previously, this can be solved by technology upgrades (DRM), but now equipped with high literacy about new media; the violations of the updated technology can be done effortlessly. Hence the balance of industry versus consumer interest is a difficult one for policymakers and regulators to balance. Intuitively, it may be that at the nascent stage of growth of a sector, regulators may choose to favor industry and reap the consequent economic benefits that are accrued. When growth becomes mature, consumer interests may begin to take priority so that society may equitably benefit from IDM.

Hence, we suggest "a layered cake model" (Mahizhnan, 2009; Waltermann and Machill, 2000) for policy makers to enter into an arrangement of coregulation with the cooperation with industry and consumers. Co-regulation necessitates that all players come together so that the industry can grow from nascent to mature, not stifle the growth and innovation it is capable of. The joining together (for a defined mutual benefit) of policy makers (who set the rules), regulators (who enforce a level playing field), consumer advocacy groups (who watch out for consumer interests and educate them of their rights), and industry forums or trade associations (who represent the technology and business interests of IDM producers and providers) in order to agree and promote the balance between various tension points can grow an IDM sector or market from nascent to mature while not stifling growth and innovation.

\section{Distribution: Business Models and Frameworks as a Basis for Market Growth}

Both the statistical tests and expert validation workshops emphasized the idea that the innovation in business models is vital for the sustainable development and breakthrough growth among three sectors. This serves as a reminder to policy makers of their role in creating a business-friendly environment and enforcing regulations transparently.

Policy makers should proactively participate in the understanding of business models and balance the interests of suppliers (industry) and consumers, as an arms-length but interested party. Policy makers may also play an objective role in the process and make innovative new models more sustainable (perhaps by actively policing the effect of cross-subsidies, 
monopolistic pricing or barriers to entry). Based on that, new standards and regulations can be made to mark the boundary of each part and clarify the responsibility and obligation of players.

More specifically, the online music industry where the policy making institutions did not work out very well, is an example. The entry of the hardware suppliers such as Apple, Nokia has transformed the disadvantageous situation for music principals that are at the heart of creativity. (Unclear) This is a lesson for policy makers that any business models related to policy making should fully consider the unprecedented development of the industry and the underground power of the new entrants.

A sustainable and stable business model can only be achieved by truly understanding the needs of producers, consumers or even prosumers. All three sectors tracked in this research are characterized by high levels of interaction and end-user-design capability, which posed a serious challenge to traditional content providers. Policy makers should leverage this influence to keep a balance among traditional players and new entrants as well as duo-players, that is, prosumers. The role of policy makers in this regard is to fit the corresponding policies into society's need for them. It is dynamic and evolving.

\section{Innovation: Social Networks and Network Policy as a Basis for Cooperation}

Interaction is a major common characteristic among online music, IPTV and MMORPG. From the previous analysis, it is known that network policy ranks at the very top of events and triggers. In the five clusters derived from the Multi-Dimensional Scaling (MDS) statistical procedure, the closeness between network policy and end-user design is evident. Policy makers should put these two points together. Moreover, the net neutrality issue is still under the limelight for its role in promoting innovation and freedom. Its pros and cons have been explored extensively (Mahizhnan, 2009; Meisel \& Sullivan, 2002; Papagiannidis et al., 2008) but it is clear that the simultaneous impact of social network and network policy is also closely related to business models.

In the MMORPG sector, numerous user-generated avatars have been put into the market by industry players, user design features incorporated into devices, and customization of the platform allowed. This is another instance of a successful and sustainable business model where the profit of selling the user-generated inputs may be shared by the consumer designer, the platform provider (game supplier) and the third party (usually as money transactions). This can be applicable for user generated music and movie clips as well. However, although YouTube, MySpace, etc. have quickly emerged as very popular, the industry has not gained much profit from such innovation yet. This 
could be because, unlike MMORPG, online music is typically in an open platform with billing and payment becoming entwined with complex digital rights management protocols. So for the MMORPG sector, the win-win scenario will be one with revenue sharing within a content charging system.

For IPTV, though a wall-garden platform is on the one hand too stringent to allow entrants like consumers to compete; on the other hand, the usergenerated movie clips / videos per se are still at a nascent stage and not yet ready to monetize. Thus the IPTV providers cannot use the same revenue sharing and content charging model as that of the music. Therefore, we find that the cooperation between technology suppliers and content suppliers is a decisive factor, because one of the major difficulties is lack of premium content and unstable technology. Technology suppliers and network operators and ISPs are new entrants to this traditional content based TV industry and play a more important role in its growth. Cooperation between traditional players and new entrants becomes imperative. Thus, for the IPTV sector, we think this kind of cooperation will lead to a win-win scenario.

For MMORPG, which is the most profitable and fastest growing sector among the three, the current situation is already a win-win scenario. However, there remain some pertinent issues to be cautious about. One of them is the high switching cost of shifting among different gaming platforms. The solution is still cooperation among game development companies. But this is difficult to accomplish, as platforms are unique selling propositions. Shifting platforms may lose consumers and then the revenue may drop, so the industry uses price as a major inhibitor to counter that. However, this tactic may harm the interest of game players in the long run and may lead to a shrinking market. Therefore, for MMOPRG, we surmise that based on the current sustainable business model, the industry could yet find a way to inter-operate with each other (perhaps through an industry standard interface for games devices and applications) and expand the market pie rather than prevent each other to keep their own market share.

For online music, the initial appearance of digital music and free downloading and sharing is a zero-sum game. The content providers have persecuted customers while the latter continues to lose market share and revenue as well. Moreover, new entrants stiffen the competition yet also bring a fresh business model for the online music industry. The iTunes revenue sharing model provides a glimpse into a wall-garden style win-win scenario for multiple parties.

To sum up, social network is a double-edged sword. On the one hand, social media is a good way for industry players to find their target audience as well as market related products. On the other hand, digital products can be distributed and evaluated instantly among consumers so that it is hard to replicate (or repudiate) the reputation and trust (or lack of) in digital markets. 
Business competitors can take advantage of it to spread negative reviews against others. Therefore, policy makers should be cautious about the developmental trends of social network and guide industry players in creating a level playing field.

\section{Concluding Remarks}

It is the intended contribution of this paper to present the results of an approach to SP as a means to understand the phenomenon of IDM applications. In so doing, we may map a route to understanding the technological and social changes that accompany this emerging future of digital natives and their consumption of media and entertainment. From the automated tracking and analysis, we derived strands that were found to be related to content, consumers, distribution and service issues. Extrapolating among the Tension Points, we may suggest the boundaries of win-win versus win-lose possibilities. These are in effect the validated scenarios that emerged from the expert workshops.

To recap, in light of the discussion and synthesis in the preceding chapter, it is conjectured that three categories of scenarios may be derived from the synthesized approach to SP: 1. Win-Win Scenarios (a fair and sustainable growth regime where all the players gain from an enlarged market size), 2. Win-Lose \& Lose-Win Scenarios (an unduly competitive, winner-takes-all, zero-sum game regime where the market does not grow and is re-apportioned among the players), 3. Lose-Lose scenarios (reduction to a classic Prisoners' Dilemma regime). The implication of policy formulation will hence be derived in order to achieve win-win scenarios and avoid lose-lose scenarios.

It may be overly simplistic to define pre-conditions for market growth (in which both consumers as well as producers of IDM services win) and scenarios in which one party loses and therefore spoils the market (as the protracted IP struggle is proving to being the digital media world). However, the hybrid approach that has been adopted here does show that there are some key (and volatile) Dimensions of Analysis which are poised to influence the future more than other (less significant) ones. Innovation, distribution and intellectual property rights have repeatedly occurred in our analyses as strands that will influence the future development of the IDM industry. Regulators, industry players and consumers must be aware of this. Business models relate to almost all the other Dimensions of Analysis and Tension Points, while enduser design, which comprises of many aspects of usability top the list of significant issues. On the basis of this argument, it is concluded that a win-win scenario is given by the confluence of business models affecting producers and end-user design that affords consumers what they want. 
From the outcome of the SP exercise carried out in three sectors of the IDM marketplace, it is also apparent that policy makers and regulators play an active part in evolving the rights of consumers so that they are protected while at the same time encouraging industry growth. Technology in the form of pervasive networks (3.75G, WiMax) and ubiquitous devices (smart-phones, Tablet PCs) will keep the pressure on both consumers as well as producers of IDM services to evolve socially. Such is the interplay of technological developments and social change in the arena of IDM. Otherwise, the economic and technology clashes between the two primary players is destined to slip into a win-lose zero-sum game and further self-destruct to a lose-lose scenario. Policy makers and regulators must be mindful of these factors as they mitigate the rights of producers (industry) with the aspiration of consumers (society at large). It is conjectured that an open, competitive IDM marketplace with performance safeguards may serve both and lead to a win-win scenario. While there are differences among IDM sectors, there are minor and unified approaches to regulation and policy would be effective. Likewise, while there are differences in the hot issues derived from news and blogs, they were also not statistically significant.

\section{Acknowledgements}

This work was supported by National Research Foundation of Singapore's Interactive Digital Media R\&D Program, under research Grant NRF.2007 IDM-IDM00276. It is part of the activities of the Special Interest Group on Interactive Digital Enterprise led by Dr. Ravi S. Sharma. Many thanks are due to Messrs Goh Kheng Wee and Foo Ji Haw of Nexlabs Inc for the assistance with the Tredicate tool. Ms Yang Yi was the Research Assistant who performed the experiments and compiled the results as part of her graduate dissertation. The authors are grateful to Professors Pedro Velaro-Mora, Ringgo Ho and Xu Hong for their kind assistance with the statistical methodology and analysis.

\section{References}

Amit, R. and Zott, C. (2001) Value creation in e-business, Strategic Management Journal, 22(6-7), 493-520.

Ang, C.S., Zaphiris, P. and Mahmmod, S. (2007) A model of cognitive loads in massively multiplayer online role playing games, Interacting with Computers, 19(2), 167-179.

Berman, S. (2007) New business models for the new media world, Strategy \& Leadership, 35(4), 23-31. 
Boaventura, J.M.G. and Fischmann, A.A. (2008) Is your vision consistent? A method for checking, based on scenario concepts, Futures, 40(7), 597-612.

Bouwman, H., Meng, Z., van der Duin, P. and Limonard, S. (2008) A business model for IPTV service: a dynamic framework, Info, 10(3), 22-38.

Burt, G. (2007) Why are we surprised at surprises? Integrating disruption theory and system analysis with the scenario methodology to help identify disruptions and discontinuities, Technological Forecasting and Social Change, 74(6), 731-749.

Castronova, E. (2005a) Synthetic World: The Business and Culture of Online Games, Chicago and London: University of Chicago Press.

Castronova, E. (2005b) Worlds to Conquer Online: Multiplayer Gaming Comes of Age Synthetic Worlds: The Business and Culture of Online Games, Chicago, IL: University of Chicago Press.

Chermack, T.J. (2005) Studying scenario planning: theory, research suggestions and hypotheses, Technological Forecasting and Social Change, 72, 53-68.

Chuang, Y.C. (2006) Massively multiplayer online role-playing game-induced seizures: a neglected health problem in internet addiction, Cyber Psychology \& Behavior, 9(4), 451-456.

Coles, A.M., Harris, L. and Davis, R. (2004) Is the party over? Innovation and Music on the Web. Information, Communication and Ethics in Society Information, 2(1), 21-30.

Cornelius, P., Putte, A.V.D. and Romani, M. (2005) Three decades of scenario planning in shell, California Management Review, 34(2), 335-387.

Craig, K. (2006) Second Life Land Deal Goes Sour, Retrieved on 21 June 2010 from http://www.wired.com/print/gaming/virtualworlds/news/2006/05/70909.

Duchene, A. and Waelbroeck, P. (2006) The legal and technological battle in the music industry: information-push versus information-pull technologies, International Review of Law and Economics, 26(4), 565-580.

Duh, R., Jamal, K. and Sunder, S. (2001) Control and assurance in e-commerce: privacy, integrity and security at eBay, Sloan Management Review, 43(1), 17-35.

Eastwood, G. (2007) The Future of TV: The Evolving Landscape of HDTV, IPTV and Mobile TV, Gartner Report.

Erman, B. and Matthews, E.P. (2008) Analysis and realization of IPTV service quality, Bell Labs Technical Journal, 12(4), 195 - 212.

Fabbe, C.N., Roussat, C. and Colin, J. (2011) Future sustainable supply chains: what should companies scan? International Journal of Physical Distribution and Logistics Management, 41(3), 228-252.

Fahey, L. and Randall, R.M. (1998) Learning from the Future. London: Wiley \& Sons.

Georgantzas, N.C. and Acar, W. (1995) Scenario-Driven Planning: Learning to Manage Strategic Uncertainty, Westport CN: Greenwood Publishing.

Godet, M. (2006) Creating Future Scenario Planning as a Strategic Management Tool, Gerber, A., Radford, K., (Trans. 2nd ed.), Paris: Economica Ltd.

Griffith, M.W. and Taylor, B. (1996) The future for multimedia- the battle for world dominance, Long Range Planning, 29(5), 18-29.

Griffiths, M.D., Davies, M.N.O. and Chappell, D. (2004) Demographic factors and playing variables in online computer gaming, Cyber Psychology \& Behavior, 7(4), $479-487$. 
Guion, L.A. (2010) A 10-step process for environmental scanning, Journal of Extension, 48(4) 30-35.

Hsu, C.L. and Lu, H.P. (2007) Consumer behavior in online game communities: a motivational factor perspective, Computers in Human Behavior, 23(3), 1642-1659.

Huss, W.R. and Honton, E.J. (1987) Scenario planning: what style should you use? Long Range Planning, 20(4), 21-30.

IFPI (2005a) Digital Sales Triple to 6\% of Industry Retail Revenues as Global Music Market Falls 1.9\%, Retrieved on 30 June 2010 from www.ifpi.org/site-content/press/ 20051003.hitml.

IFPI (2005b) Recording Industry World Sales 2005, Retrieved on 04 July 2010 from www.ifpi.org/statistics/worldsales.htm.

IFPI (2008) Digital Music Report 2008, Retrieved on 15 July 2010 from http://www.ifpi. org/content/section_resources/dmr2008.html.

ITA (2005) Cyber-Sabre Provokes Real Death, 31 March, Retrieved on 30 July 2010 from

http://australianit.news.com.au/articles/0,7204,12711503\%5E15404\%5E\%5Enbv\%5 E15306-15322,00.html.

Jobs, S. (2008) Thoughts on Music, Retrieved on 30 July 2010 from http://www.apple. com/hotnews/thouthsonmusic.

Karakas, F. (2009) Welcome to world 2.0: the new digital ecosystem, Journal of Business Strategy, 30(4), 23-30.

Krogfoss, B., Sofman, L. and Agrawal, A. (2008) Caching architectures and optimazation strategies for IPTV networks, Bell Labs Technical Journal, 13(3), 16-24.

Krippendorff, K. (2004) Content Analysis: An Introduction to Its Methodology, 2nd Edition, Thousand Oaks: Sage Publication.

Kuesk, D. (2008) Cycles in Music, Retrieved on 25 Oct 2009 from http://www. futureofmusicicbook.com/2008/10/19cycles-in-music.

Kunze, O. and Li, W.M. (2007) Consumer adoption of online music services the influence of perceived risks and risk relief strategies, International Journal of Retail \& Distribution Management, 35(11), 16.

Lo, S.K., Wang, C.C. and Fang, W. (2005) Physical interpersonal relationships and social anxiety among online games players, Cyber Psychology \& Behaviour, 8(1), 15-20.

Lucchi, N. (2007) The supremacy of techno-governance: privatization of digital content and consumer protection in the globalized information society, International Journal of Law and Information Technology, 15(2), 192-225.

Marcel M. (eds.) (2000) Protecting our children on the internet, Gutersloh: Bertelsmann Foundation Publishers.

Mahizhnan, A. (2009) Saviours and barbarians at the gate: dilemmas of regulatory policies for new media, Keynote address at $2^{\text {nd }}$ SIGIDE workshop on IDM, University of Liepzig, Germany, 19- 20 September 2010.

Mantel, N. (1967) The detection of disease clustering and a generalized regression approach, Cancer Research, 27(2), 209-220.

McCaslin, M. and Burross, H.L. (2011) Research on individual differences within a sociocultural perspective: co-regulation and adaptive learning, Teachers College Record, 113(2), 325-349. 
Meisel, J.B. and Sullivan, T.S. (2002) The Impact of the Internet on the law and economics of the music industry, Info, 4(2), 6-21.

Millett, S.M. (2003) The Future of scenarios: challenges and opportunities, Strategy \& Leadership, 31(2), 16-24.

Molteni, L. and Ordanini, A. (2003) Consumption patterns digital technology and music downloading, Long Range Planning, 36(4), 18-32.

Norusis, M.J. (2008) SPSS Statistics 17.0 Advanced Statistical Procedures Companion, Upper Saddle River, NJ: Prentice Hall.

Ogilvy, J.A. (2002) Creating Better Futures: Scenario Planning as a Tool for a Better Tomorrow, New York: Oxford University Press.

Pagani, M. (2009) Road-mapping 3G mobile TV: strategic thinking and scenario planning through repeated cross-impact handling, Technological Forecasting and Social Change, 76(3), 382-395.

Papagiannidis, S., Bourlakis, M. and Li, F. (2008) Making real money in virtual worlds: MMORPGs and emerging business opportunities, challenges and ethical implycations in metaverses, Technological Forecasting \& Social Change 75, 610-622.

Pereira, F. (2004) The Future of Switched Voice: A Firecracker Approach, Centre for Technology Management, Los Angeles: USC Press.

Poel, M., Renda, A. and Ballon, P. (2007) Business model analysis as a new tool for policy evaluation: policies for digital content platforms, Info, 9(5), 86-100.

Rajamma, R.K., Paswan, A.K. and Ganesh, G. (2007) Services purchased at brick and mortal versus online stores and shopping motivation, Journal of Services Marketing, 21(3), $200-213$.

Reedy, S. (2008). IPTV ADDS TO THE BUSINESS PLAN. Telephony, 249(1), 16-18.

Rikkonen, P. and Tapio, P. (2009) Future prospects of alternative agro-based bioenergy use in Finland-constructing scenarios with quantitative and qualitative Delphi data, Technological Forecasting and Social Change, 76(7), 978-990.

Rongone, A. and Turconi, A. (2003) The television revolution within the multimedia convergence: a strategic reference framework, Management Decision, 41(1), 48-71.

Sharp, C.E. and Rowe, M. (2006) Online games and e-business: architecture for integrating business models and services into online games, IBM Systems Journal, 45, 161-181.

Shin, D.H. (2005) Technology convergence and regulatory challenge: a case from Korean digital media broadcasting, Info, 7(3), 47-58.

Shin, D.H. (2007) Socio-technical analysis of IPTV: a case study of Korean IPTV, Info, 9(1), 66-79.

Shoemaker, P.J.H. (1991) When and how to use scenario planning; a heuristic approach with illustration, Journal of Forecasting, 10(6), 549 - 565.

Schwartz, P. (1991) The Art of the Long View, New York: Doubleday.

Shell (2005) Scenarios, Retrieved on 30 July 2010 from http://www.shell.com/ home/content/aboutshell/our_strategy/shell_global_scenarios/dir_global_scenarios_0 7112006.html.

Spero, I. and Stone, M. (2004) Agents of change: how young consumers are changing the world of marketing, Qualitative Market Research, 7(2), 155-159.

Swatman, P., Krueger, C. and van der Heijden, K. (2006) The changing digital content landscape an evaluation of e-business model development in European online news 
and music, Internet Research, 16(1), 53-82.

Szulansk, G. and Amin, K. (2004) Disciplined imagination: strategy making in uncertain environments, in Day. G., Shoemaker, P. and Gunther, R. (Eds.), Wharton on Managing Emerging Technologies, 189-205, New York: John Wiley \& Sons.

Torgerson, W.S. (1965) Multidimensional scaling of similarity, Psychometrik, 30(4), 127-145.

Tychsen, A., Hitchens, M., Brolund, T. and Kavakli, M. (2006) Live action role-playing games control, communication, storytelling, and MMORPG similarities, Game and Culture, 1(3), 252-275.

van der Heijden, K. (2005) Scenarios: The Art of Strategic Conversation (2nd ed.), London: John Wiley.

Wack, P. (1985a) Scenarios: shooting the rapids, Harvard Business Review, 63(6), 139151.

Wack, P. (1985b) Scenarios: uncharted waters ahead, Harvard Business Review, 63(5), 73-90.

Walsh, G., Mitchell, V.W., Frenzel, T. and Wiedmann, K.P. (2003) Internet-induced changes in consumer music procurement behavior: a German perspective, Marketing Intelligence \& Planning, 21(5), 305-318.

Waltermann, J. and Machill, M. (eds.) (2000) Protecting our children on the internet, Gutersloh, Germany: Bertelsmann Foundation Publishers.

Williams, D. (2006) Groups and goblins: the social and civic impact of an online game, Journal of Broadcasting and Electronic Media, 50(4), 651-670.

Williams, R. and Cothrel, J. (2000) Four smart ways to run online communities, Sloan Management Review, 41(4), 81-91.

Yans, C. (2004) Virtual property brokers promise 'We deliver instantly 24 hours a day', Retrieved on 30 Aug 2009 from http://www.cgonline.com/content/view/21/40/.

Young, F.W. (1999) Multidimensional Scaling, Retrieved on 23 Dec 2010 from http://forrest.psych.unc.edu/teaching/p208a/mds.html.

Young, F.W. and Valero M.P. (2006) Visual Statistics: Seeing Data with Dynamic Interactive Graphics, New York: Wiley-Inderscience.

Zwicky, F. (1969) Discovery, Invention, Research-Through the Morphological Approach, Toronto: Macmillan 